\title{
Stimulanzien vertragen sich mit Alkohol
}

Die Aufmerksamkeitsdefizit-/Hyperaktivitätsstörung (ADHS) ist häufig mit anderen Komorbiditäten vergesellschaftet, unter anderem mit Substanzmissbrauch. Deshalb ist es wichtig zu wissen, ob es Wechselwirkungen zwischen Stimulanzien und harten oder weichen Drogen gibt.

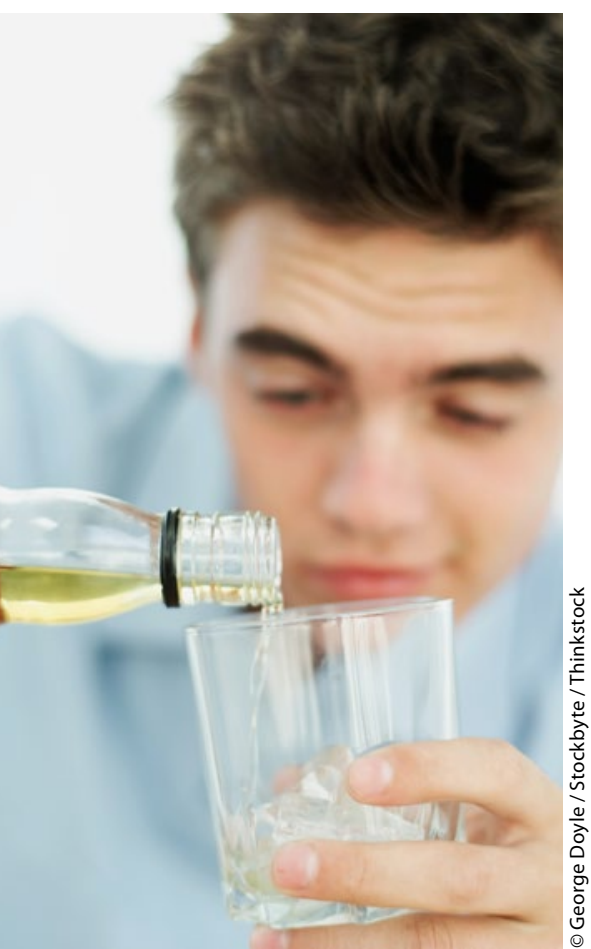

Bei Jugendlichen mit ADHS ist aufgrund einer schwachen Impulskontrolle häufig nach einem Glas nicht Schluss.
$\mathrm{D}$ e Autoren führten eine systematische Literaturrecherche über mögliche gefährliche pharmokologische Interaktionen bei einem zeitgleichen $\mathrm{Ge}$ brauch von Stimulanzien und Alkohol beziehungsweise anderen Drogen durch. Medline, EMBASE und PsychINFO wurden über den Zeitraum von 19922014 nach entsprechender Literatur durchsucht. 20 Artikel, die die Eingangskriterien erfüllten, wurden ausgewertet: Stimulanzien, in therapeutischen Dosen eingenommen, führten in Kombination mit Alkohol zu keinen gefährlichen $\mathrm{Ne}$ benwirkungen. Bei der Kombination von Stimulanzien und Kokain kann es zu einem Verlust der euphorischen Wirkung des Kokains kommen sowie zu verstärkter Traurigkeit.

Barkla XM et al. Are there any potentially dangerous pharmacological effects of combining ADHD medication with alcohol and drugs of abuse? A systematic review of the literature. BMC Psychiatry 2015;15:270

\section{Kommentar}

Eine oft gestellte Frage - vor allem der Eltern von Jugendlichen mit ADHS - in der
Praxis lautet: Darf mein Sohn/meine Tochter abends Alkohol trinken trotz Einnahme von Retardpräparaten von Methylphenidat oder auch Amphetaminsulfat? Meist fragen die Eltern auch suggestiver: „Er darf doch nicht abends Alkohol trinken, oder?" Nicht nur wir Ärzte müssen vermeiden (um nicht unsere Glaubwürdigkeit zu verlieren), der verlängerte pädagogische Arm der Eltern zu sein. Vielmehr gibt es keine Rationale, warum der mit Stimulanzien behandelte Jugendliche mit ADHS abends keinen Alkohol trinken sollte. Natürlich gibt es sicher viele andere Gründe dafür, einen regelmäßigen und auch übermäßigen Alkoholkonsum zu vermeiden.

Auch wenn die Studienlage dürftig ist, so weisen die wenigen vorhandenen Untersuchungen darauf hin, dass nicht die Medikation ein Hindernis für einen abendlichen Alkoholkonsum ist. Bei Jugendlichen mit ADHS besteht vielmehr ein zusätzliches Risiko der Impulskontrollschwäche, das dazu führen kann, dass eben nach einem Glas nicht Schluss ist, sondern der Alkoholkonsum grenzenlos wird. Eine bewährte Empfehlung von mir lautet also eher: dem Jugendlichen mit ADHS bei abendlichen Ausgängen noch eine zusätzliche Abenddosis mit Stimulanzien zu verabreichen. Der Jugendliche hat dann eine bessere Kontrolle darüber, wie viel er trinkt - und nicht selten ist dann sogar er derjenige, der seine Freunde nach Hause bringt!

Dr. Kirsten Stollhoff

\section{Schlafprobleme und selbstverletzendes Verhalten treten häufig gemeinsam auf}

Zu den Risikofaktoren für selbstverletzendes Verhalten gehören Umwelt- und psychologische Faktoren. Ob bei Jugendlichen ein Zusammenhang zwischen spezifischen Schlafproblemen und selbstverletzendem Verhalten besteht, untersuchte nun eine Gruppe von Wissenschaftlern aus Norwegen.

S elbstregulatorische Prozesse und insbesondere die Schlafregulation scheinen eine bedeutende Rolle im Rahmen von selbstverletzendem Verhalten $\mathrm{zu}$ spielen. Schlafprobleme erwiesen sich dabei als von psychischen Erkrankungen unabhängige Prädiktoren für
Selbstverletzung und Suizid. Ob spezifische Ausprägungen von Schlafproblemen das Auftreten von selbstverletzendem Verhalten beeinflussen, ist bislang unklar.

Im Rahmen einer norwegischen, populationsbasierten Studie zur seelischen
Gesundheit wurden 10.220 Jugendliche zwischen 16 und 19 Jahren zu selbstverletzendem Verhalten und Schlafproblemen befragt. Dabei wurde zwischen Insomnie im Sinne von Schwierigkeiten beim Ein- oder Durchschlafen, im Bett verbrachter Zeit, Einschlafzeit sowie Erwachen nach Einsetzen des Schlafs unterschieden. Zudem wurden demografische Daten, Symptome einer Depression, Perfektionismus und Symptome eines ADHS erhoben.

Die Teilnehmerquote lag bei $53 \%$, $98 \%$ der Befragten waren noch Schüler. $7,2 \%$ berichteten von selbstverletzendem Verhalten, mehr als die Hälfte von ihnen $(55 \%)$ hatten bereits zwei oder 